\title{
Progrès technique, changement dans la société et développement des grands systèmes techniques
}

In: Flux n²2, 1995. pp. 11-16.

\begin{abstract}
The development of large technical infrastructure systems, changes in the technology pool, and changes in societal governance are closely interrelated. Political and economic structures shaped the emerging modern traffic and communication systems, which in turn facilitated the growth of the strong modern state. Today, with further technological advances, we observe parallel changes from hierarchical to network forms in society and in large technical systems.

\section{Résumé}

Le développement des grands réseaux d'infrastructure, les changements technologiques et les évolutions des modèles de gouvernance sont fortement interdépendants. Les structures politiques et économiques ont donné leur forme aux systèmes modernes de circulation et de communication, qui à leur tour ont facilité la montée en puissance de l'Etat fort moderne. Sous l'effet de nouveaux progrès techniques, on assiste aujourd'hui dans la société et dans les grands systèmes techniques à une évolution parallèle de structures hiérarchiques vers des formes d'organisation en réseau.
\end{abstract}

Citer ce document / Cite this document :

Mayntz Renate. Progrès technique, changement dans la société et développement des grands systèmes techniques. In: Flux $\mathrm{n}^{\circ} 22$, 1995. pp. 11-16.

doi : 10.3406/flux.1995.1043

http://www.persee.fr/web/revues/home/prescript/article/flux_1154-2721_1995_num_11_22_1043 


\section{FLUX}

$n^{\circ} 22$

Octobre - Décembre

1995

pp. $11-16$

Progrès technique,

changement dans la société et développement des grands systèmes techniques

Renate MAYNTZ, B.A. de Wellesley College (USA) est docteur en sociologie de la Freie Universität Berlin et docteur honoris catusa des Universités d'Uppsala et de Paris. Elle a occupé une chaire à la Freie Universität Berlin et à la Hochschule fiir Verwaltungsuissenschaften à Spire et enseigné à Columbia University à la New School for Social Research à New York, à l University of Edinburgh, à la FLASCO (Facultad Latin-Americana de Cienzas Sociales) de Santiago du Chili, et à Stanford University. Alant de devenir Directrice du Max-PlanckInstinu fiir Gesellschaftsforschung de Cologne, le Dr Mayms occupait une chaire à l'Université de Cologne et était Directrice de l'Institut fiar angewandte Sosialforschung. Elle occupe toujours un poste homorifique a lWniversité de Cologne. Ses principanx intérêts dans la Recherche: la macro-therorie sociologique. la recherche comparative sociale et politique. la sociologie

arganisationnelle el administrative ef la soriologie de la technologie. Avec Thomas P. Hughes, elle est co-éditrice de The Development of Large

Technical Systems (Frankfurt a.M. :

Campus, 1988).
$\mathrm{L}$ E TERME "grand système technique (GST)" est utilisé aujourd hui pour désigner au moins trois classes différentes de phénomènes empiriques : des programmes complexes tel que le projet Manhattan qui a produit la bombe atomique, des installations telles que des centrales nucléaires, et des systèmes d'infrastructures tels que les réseaux de chemin de fer, d'électricité ou de téléphone. Les trois types de GST sont des éléments importants du processus général de développement technologique. De plus, ils peuvent être liés : un GSTprogramme peut aboutir à un GST-installations techniques, et celui-ci à son tour faire partie d'un système d'infrastructures - par exemple, la centrale nucléaire fait partie du système électrique. Malgré ces relations complexes, je parlerai uniquement des grands systèmes techniques d'infrastructures et je n'utiliserai le terme GST que dans ce sens restreint.

Mon approche dans cet article sera macro-sociologique. Cette perspective est différente de l'approche d'études de cas qui prédomine dans la littérature GST. Mon point de départ est l'hypothèse que les GST sont un moteur important du changement de la société en général. Ceci sera développé dans la première partie de l'exposé. Ensuite j'essaierai de résumer ce que nous avons appris d'une manière générale sur les dynamiques de la croissance des GST. Dans la dernière partie, je débattrai sur les liens entre le développement des grands systèmes techniques d'infrastructures et les changements dans la gestion de la société.

\section{GST EN TANT QUE SOUS-SYSTÈMES DANS LA SOCIÉTÉ}

Les grands systèmes techniques d'infrastructure sont historiquement des phénomènes récents. même si les fonctions qu'ils exerçaient - transport, communication, distribution d'énergie - correspondent à des besoins humains fondamentaux qui ont été satisfaits d'une façon ou d'une autre dans les sociétés plus anciennes. Les professions et les petites organisations qui s`occupent de telles activités d'infrastructure existaient même avant l'accélération du développement technologique du début du 19ème siècle. Mais, c'est seulement avec la production d'énergie à grande échelle, avec le chemin de fer. l'avion et le téléphone. que ces différents champs d’activité sont devenus des systèmes fonctionnels caractérisés par une grande taille. une productivité élevée et une organisation structurée.

Cette communication est basée en grande partie sur un article précédent en allemand (Marnt: 1993). 
Tous les sous-systèmes fonctionnels de la société, en particulier les activités économique et militaire de l'Etat. utilisent aujourd'hui des objets techniques sophistiqués comme ressources. Les systèmes modernes d'infrastructures ne font pas qu'utiliser, mais sont en fait essentiellement fondés sur des innovations techniques spécifiques.

Sans l'invention du groupe électrogène et de l'ampoule, les "réseaux électriques" (Hughes 1983) n'auraient pas existés. La machine à vapeur a joué le même rôle pour le chemin de fer, le télégraphe et le téléphone pour les télécommunications modernes. Les systèmes d'infrastructure modernes sont donc fondés sur la technologie d'une façon très directe, et ils diffèrent en cela des autres sous-systèmes fonctionnels de la société tel que l'économie, le régime politique, ou le système de santé.

La dépendance technologique des GST ne signifie pas, cependant, que leurs caractéristiques institutionnelles et organisationnelles sont déterminées par la technologie qui leur sert de base. La technologie est une contrainte pour les choix organisationnels, mais il y a normalement plusieurs alternatives possibles. Toute technologie offre des opportunités diverses (quoique généralement en nombre limité). Ceci a été observé et discuté par des sociologues industriels depuis longtemps, en commençant par la célèbre étude de Tavistock sur les méthodes d'exploitation des mines de charbon (Trist \& Banforth 1951 ; Trist et al. 1963). Sur une plus grande échelle, on peut observer une certaine corrélation entre la technologie disponible et l'organisation des GST, en comparant la forme institutionnelle des réseaux électriques ou des systèmes de télécommmunication dans différents pays, comme l'ont fait respectivement Thomas Hughes (1983) et Volker Schneider (1995). La relation contingente entre la technologie et l'organisation ouvre la voie pour l'étude de l'influence des facteurs sociaux, politiques et culturels.

Les systèmes d'infrastructure sont, par leur nature même, des systèmes de support, mais cela ne veut pas dire qu'ils n'ont joué qu'un rôle mineur dans le développement de la société moderne. D'ailleurs, on peut raisonnablement penser que l'effet général des GST sur le processus de développement social a été plus fort que l'effet des technologies industrielles, médicales ou autres. Les télécommunications modernes et les média ont fortement influencé les formes de la décision politique. Sans les systèmes modernes de production d'énergie, de transport et de télécommunications, la révolution industrielle n'aurait pas eu lieu, et la croissance économique que nous avons connue depuis cent ans n'aurait pas été possible. Comme l'a démontré James Beniger, l'invention de la machine à vapeur et son utilisation directe dans la production de mar- chandises n’a pas été le principal facteur de la révolution industrielle. Le facteur décisif a été l'amélioration des conditions de transport grâce aux paquebots et au chemin de fer, pour laquelle la machine à vapeur a été utilisée intensivement. Plus tard, les télécommunications modernes ont permis de résoudre les problèmes de coordination générés par le volume croissant, la vitesse, et l'expansion spatiale des flux de matières premières, de marchandises et de capitaux (Beniger, 1986). Les récents changements structurels de l'économie - la globalisation, la sous-traitance et la production en juste-à-temps - reposent également sur les télécommunications modernes. Sans elles, les marchés financiers modernes ne pourraient pas fonctionner, et les multinationales ne pourraient pas coordonner leurs établissements dispersés à travers le monde.

L'efficacité des systèmes modernes d'infrastructure a beaucoup amélioré le fonctionnement des sous-systèmes économiques, politiques, et sociaux. Mais la médaille a son revers. Une augmentation de l'efficacité des GST implique souvent un risque plus important en cas d'accident. Plus encore, cependant, il faut souligner la forte dépendance qui résulte de l'utilisation des systèmes techniques d'infrastructures. Ces systèmes s'introduisent, pour ainsi dire, dans tous les sous-systèmes fonctionnels (qu'ils soient économiques, militaires ou médicaux). Cela aboutit souvent à une dépendance complète et déséquilibrée vis-àvis d'un seul système technique ; en cas de défaillance, il n'est pas facile de trouver un substitut. La régression fonctionnelle est souvent la seule porte de sortie dans un cas pareil - par exemple utiliser des bougies et, si on a la chance d'en avoir une, une cheminée en cas d'une défaillance importante du système électrique. Puisque les systèmes d'infrastructures répondent à des besoins fondamentaux, il est impossible d'éviter cette dépendance en rationnant volontairement la demande. En bref, les utilisateurs d'un GST n'ont pas d'alternative. La possibilité de protester n'est sur ce plan d'aucun secours. Au contraire : les critiques faites par les utilisateurs d'un GST entraînent des améliorations qui renforcent encore le système. Le risque de conséquences catastrophiques en cas de défaillance d'un GST est donc devenu menaçant. au point de dominer le débat actuel sur le développement technologique.

\section{LES DYNAMIQUES DE DÉVELOPPEMENT DES GST}

En raison de leur origine technique, les systèmes d'infrastructure modernes obéissent à un modèle de développement spécifique. Ceci est vrai à la fois au macro- 
niveau du développement social et pour chaque système d'infrastructures. Le macro-processus consiste en la multiplication des GST qui fournissent parfois les mêmes services, mais réalisent le plus souvent des fonctions nouvelles. Le moteur de ce processus est l'innovation scientifique et technologique stimulée par la curiosité. La logique propre de la technologie est exprimée au mieux dans l'image de l'homo faber qui veut tester les limites des capacités humaines - que ce soit pour voler, pour communiquer à grande distance, pour aller sur la lune, pour créer de nouveaux animaux ou plantes, etc. Cela crée continuellement des opportunités pour le développement de nouveaux GST. Le transport aérien commercial est un des GST les plus récents. Actuellement, on assiste dans un certain nombre de pays à l'émergence d'un système nucléaire - qui n'est pas seulement constitué des centrales nucléaires, mais de l'interconnexion organisée et contrôlée des producteurs de combustible, des centrales nucléaires, de la gestion des déchets nucléaires, des instituts de recherche, et quelquefois également des institutions militaires (Rochlin 1992).

En dehors de tels GST de premier ordre se développant autour d'une technologie spécifique principale, on observe également le développement de GST de deuxième ordre. Ils fournissent les services spécifiques comme la transplantation d'organes en coordonnant de manière précise plusieurs GST de premier ordre - en l'occurence : transport aérien, transport routier, banques de données, téléphone, etc (Braun \& Joerges 1994). De cette façon, les effets d'innovations technologiques données provoquent continuellement le développement de nouveaux systèmes d'infrastructure et de services secondaires.

En ce qui concerne la croissance individuelle de chaque GST, la littérature disponible (voir surtout Mayntz \& Hughes 1988) autorise quelques généralisations. La première a trait à l'évolution structurelle des réseaux techniques. Jusqu'à maintenant, celle-ci s'est en général conformée au modèle du développement des réseaux de téléphone et d'électricité. Dans un premier temps, de multiples réseaux de petite taille sont apparus, qui se sont ensuite interconnectés en réseaux régionaux, nationaux puis transnationaux. Les premiers embryons de développement locaux sont déterminés par les considérations administratives, militaires ou économiques, selon l'intérêt du principal promoteur du GST considéré. Plus tard, les frontières politiques deviennent importantes pour la forme du réseau. Lorsque, au cours des processus d'internationalisation et de globalisation de l'économie, les réseaux nationaux deviennent internationalement intégrés, le premier modèle de développement se répète à une plus grande échelle.
Un deuxième effet, en principe plus important, a trait à l'interaction dynamique entre l'offre et la demande. Cette interaction existe dans des systèmes fonctionnels non-techniques. Commercialiser un produit signifie plus que faire concurrence avec d'autres producteurs pour avoir une part de la demande existante ; cela implique souvent en premier lieu de susciter cette demande. De la même façon, le nombre croissant de médecins contribue au développement des services de santé tant que celui qui paie pour ces services est convaincu de leur nécessité. Dans le développement du système politico-administratif également, la lutte pour le pouvoir joue un rôle analogue. Dans le cas de systèmes techniques d'infrastructures, l'aspect essentiel de l'offre est la technologie elle-même, donc nous devons examiner l'importance relative de l'offre technologique et de la demande sociale.

Les termes de l'interaction entre ces deux facteurs changent d'une façon caractéristique au cours des différentes phases du développement du GST. La demande exprimée est relativement peu importante dans la phase initiale du développement du système. Les innovations technologiques importantes, qui résultent souvent d'une recherche stimulée par la curiosité, s'apparentent à des solutions en quête de problèmes. C'est pourquoi l'on peut parler de logique de l'offre.

Au début du développement du chemin de fer, de l'électricité et du téléphone, les inventeurs et les constructeurs des premiers systèmes ont consacré d'importants efforts à une innovation technologique ingénieuse malgré le scepticisme de leur clientèle potentielle. A cette époque, la demande pour ces services est satisfaite par les systèmes existants - à des niveaux de qualité acceptés, pour un coût plus faible et avec moins de risques. Ceci était vrai pour l'éclairage électrique par rapport à l'éclairage au gaz, et c'était également vrai pour le téléphone par rapport au télégraphe électrique qui l'a précédé. La nouvelle technologie est donc souvent perçue comme un moyen pour développer et améliorer le système existant. En Allemagne, par exemple, le téléphone a d'abord été utilisé pour développer le réseau télégraphique à partir de ses terminaux locaux (Thomas 1995). Dans la phase initiale du développement des GST donc, la logique technologique de l'offre joue un rôle beaucoup plus important que la prise en considération de la demande. Ce n'est que dans une phase ultérieure que le développement du système et la croissance de la demande doivent aller de pair. Ce constat s'applique aussi aux innovations plus récentes dans le domaine télématique, telle que le vidéotexte et l'ISDN.

La croissance du système ne peut s'auto-entretenir qu'à partir d'un taux 'bénéfices sur coûts' minimal. Ceci 
concerne plus directement les services destinés à de nombreux utilisateurs privés. Dans le cas de technologies - et de systèmes techniques - qui sont jugés essentiels par l'institution militaire ou par l'administration, la demande est concentrée dans les mains de l'Etat. On peut penser aujourd'hui aux technologies spatiales et aux engins balistiques ; autrefois, c'était le télégraphe. En raison de l'utilité politique du télégraphe, les réseaux français et allemands se sont développés en tant que monopoles de l'Etat. Lorsque l'Etat, sans vouloir réserver un GST à son seul usage, considère toutefois son développement utile, par exemple pour la croissance économique, il peut le soutenir si la demande privée ne suffit pas. Ceci s'observe, par exemple, dans le cas du développement initial du chemin de fer en France et en Allemagne, où la demande du marché était insuffisante pour atteindre la phase de croissance autonome.

Pour analyser les chances de développement d'un nouveau GST, il est donc important d'étudier la constellation de parties intéressées et leurs intérêts spécifiques. Aucun GST ne peut survivre s'il n'y a pas une demande suffisante pour ses services (et une volonté de les financer). Il n'est pas nécessairement bénéfique que la demande soit concentrée dans les mains de l'Etat plutôt que dispersée entre de nombreux clients privés. Dans tous les cas, cependant, la dépendance du client (ou des clients) vis-àvis d'un GST représente sa meilleure garantie de survie.

Le développement des GST est aussi soumis à des facteurs favorables ou défavorables spécifiques. Deux obstacles, en particulier, doivent être notés. Le premier tient à l'importance des capitaux nécessaire pour construire un nouveau GST. Là où un investissement initial très élevé est nécessaire, la relation de marché entre producteurs et utilisateurs privés est insuffisante, et l'engagement de promoteurs puissants tels que des banques privées ou l'Etat paraît nécessaire. Un deuxième problème est créé par les externalités de réseau (positives) qui existent lorsque, comme dans le cas du téléphone, l'utilité du service pour l'utilisateur individuel augmente avec le nombre d'autres utilisateurs déjà branchés sur le réseau. De telles externalités du réseau positives peuvent garantir une croissance soutenue d'un système déjà bien développé, mais dans la phase initiale, lorsque le nombre d'utilisateurs est encore faible. les bénéfices individuels sont faibles également, ce qui provoque un problème de masse critique (Hohn Schneider 1991). Pour atteindre la phase de croissance autonome du réseau, il sera peut-être nécessaire d'offrir des incitations aux premiers utilisateurs - comme l'a fait l'Etat Français dans le cas du vidéotexte français Minitel afin d'atteindre le seuil critique de croissance (Schneider, Thomas, Vedel 1991).
Un moteur important du développement du système, qui semble spécifique aux GST, réside dans les défauts mêmes du fonctionnement du système, les "saillants à rebours" pour reprendre le vocabulaire de Thomas Hughes (1983). Le fait que, dans le cas des GST, les défauts favorisent le développement du système plutôt qu'ils ne l'entravent, ce fait résulte de la relation existant entre la technologie employée, l'organisation du système et la mesure de ses performances. Il est important, cependant, de distinguer entre les différents types de défauts de fonctionnement. Certains problèmes de congestion (surcharge), de mauvaise qualité ou de sécurité industrielle peuvent être résolus sans innovation. Par exemple, on peut répondre aux besoins croissants de services téléphoniques par un simple accroissement de la capacité du réseau. Mais les défauts de fonctionnement liées à la technologie utilisée plutôt qu'à la simple taille du système favorisent directement la recherche d'améliorations techniques. Un exemple dans l'histoire du téléphone est donné par la transition des premiers centraux téléphoniques mécaniques aux centraux numériques d'aujourd'hui. Ces défauts favorisant l'innovation peuvent être également des problèmes de congestion, de mauvaise qualité, ou de sécurité industrielle. Lorsque la technologie n'est pas uniquement un instrument, mais la véritable base du système, les efforts pour accroître l'efficacité se traduisent directement par des innovations technologiques. Celles-ci se répercutent alors sur les GST, selon un processus rétroactif tout-à-fait différent de ce que l'on observe pour les systèmes sociaux non techniques.

Notons que la conception de solutions technologiques pour résoudre des problèmes de nature quantitative ou qualitative peut se substituer à un changement organisationnel, A première vue, les solutions technologiques peuvent sembler "neutres", tandis que les changements organisationnels affectent de façon flagrante les différents acteurs engagés dans le système ; on se rend compte généralement trop tard que les choix techniques ont un effet analogue. Ce n'est que lorsque des améliorations techniques s'avèrent insuffisantes pour résoudre un défaut de fonctionnement donné, que l'on essaie de trouver une solution de nature organisationnelle, comme Eurocontrol a été conçu pour résoudre le problème de congestion du trafic aérien et les retards croissants qui en résultaient (Resch 1994).

\section{GST ET LA GESTION DE LA SOCIÉTÉ}

L'organisation des grands systèmes techniques d'infrastructures est étroitement liée aux formes de d'organisation de la société - une relation rarement obser- 
vée puisque la théorie macro-sociologique a eu généralement tendance à négliger les GST en tant que tels. Commençons par la relation entre le développement des GST et l'Etat. Il s'agit d'une relation de co-évolution. L'Etat moderne (19ème siècle) comme les systèmes d'infrastructures modernes (19ème siècle) ont été organisés et structurés hiérarchiquement, quoique pour des rajsons différentes. La structure hiérarchique des Etatsnations européens était une conséquence de la répartition inégale du pouvoir dans la société. conjuguée avec des considérations de contrôle politique. La forme centralisée des premiers GST a été en grande partie due aux nécessités techniques, surtout dans le cas des GST basés sur des réseaux physiques, tels que des lignes, des câbles ou des rails. Mais quoique différentes forces étaient à l'oeuvre pour les modeler, les systèmes d'infrastructures modernes et les Etats-nations européens modernes se sont renforcés mutuellement dans leur développement. Le télégraphe et le téléphone en particulier ont contribué à l'expansion d'un système centralisé d'administration publique, encourageant ainsi la hiérarchisation politique. L'Etat à son tour a facilité l'établissement de grands monopoles privés, ou a même pris en charge le développement et le fonctionnement de nouveaux systèmes d'infrastructures. Dans tous les cas, ceci a encouragé des formes hiérarchiques d'organisation. En plus, les nouveaux GST ont provoqué des interventions réglementaires répétées et ont donc renforcé le développement de l'Etat moderne réglementaire.

Les systèmes techniques d'infrastructure modernes ont également renforcé les tendances endogènes de l'industrie à la hiérarchisation. D'abord. les GST sont devenus eux-mêmes de grandes sociétés commerciales. En plus, cependant, les structures organisationnelles, les formes juridiques et les modes de financement utilisés dans la construction - et parfois inventés spécifiquement dans ce but, comme dans le cas du chemin de fer- ont fournis des modèles que l'industrie a repris par la suite.

Les tendances à la hiérachisation dans l'industrie et dans l'appareil de l'Etat se sont unis dans le complexe militaro-industriel, association entre la puissante industrie de l'armement et l'institution militaire qu'Eisenhower déconseillait déjà en son temps (Hughes 1991, 442). Pris ensemble. ces mécanismes ont augmenté la concentration du pouvoir dans la société.

Aujourd’hui, l'ancienne symbiose entre les hićrarchies politiques et industrielles (y compris les GST). est devenue précaire. Avec le temps, les effets secondaires négatifs de ces structures hiérarchiques sont devenus apparents et commencent à menacer leur pérennité. Le mécanisme de renforcement mutuel se transforme donc en un défaut commun. Le puissant Etat hiérarchique est remis en question par des mouvements de décentralisation qui se font au nom de l'autonomie locale ou régionale. Le fédéralisme de l'après-guerre en Allemagne, le nouveau régionalisme en Espagne et en Italie, la décentralisation en Grande Bretagne. et les efforts correspondants en France vont tous dans la même direction. En même temps, la capacité limitée de l'Etat central à résoudre les problèmes a entraîné une réévaluation de l'autorégulation sectorielle et des formes privées de gouvernement (voir Mayntz et Scharpf 1995). Enfin, point important, les formes hiérarchiques semblent en contradiction avec les valeurs démocratiques qui dominent aujourd'hui. Ce changement de valeurs a des répercussions sur l’armée, mais aussi sur les grandes entreprises industrielles, qui apparaissent aujourd'hui comme l'antithèse de la démocratie.

Les grands systèmes techniques, à leur tour, sont critiqués pour la concentration de pouvoir qu'ils représentent, pour leur tendance à échapper au contrôle du pouvoir politique et des consommateurs, et pour les risques énormes associés à leurs défaillances (La Porte 1991). Et de fait, l'extension des monopoles à l'échelle nationale, associée à des formes de gestion rigides et à des structures de contrôle hiérarchiques, fait que ces systèmes deviennent en effet de plus en plus insensibles aux formes externes de contrôle ; créés pour servir la société, ils en sont devenus les maîtres. Les grands systèmes techniques provoquent ainsi une sensation de malaise, et suscitent des protestations dirigées à la fois contre les systèmes industriels et contre les régimes politiques qui leur sont si étroitement liés. Ceci est bien démontré par les critiques des groupes gauchistes et écologistes contre le spectre de "l’Etat nucléaire", le raisonnement étant qu'en raison du haut risque associé à l'énergie nucléaire en cas d'accident, de sabotage ou d'action terroriste, un contrôle hiérarchique strict est inévitable. Ainsi, la réaction contre le pouvoir croissant des grands systèmes techniques, et la situation de dépendance qui en résulte pour la société, contribue à miner l'acceptation de toute forme hiérarchique de gestion.

Une attitude globalement négative envers les formes hiérarchiques de gestion, de la société en général et dans les GST en particulier, peut très bien avoir contribué aux politiques de déréglementation proposées par des gouvernements néo-conservateurs au nom des principes "moins d'Etat et plus de marché”. La déréglementation des GST organisés en monopoles implique la déconcentration verticale, ou la dé-intégration de ces organisations. Cela a eu lieu dans le cas des télécommunications. et affecte maintenant les réseaux de chemin de fer et de l'électricité. Dans le cas des réseaux téléphoniques. on s'achemine vers une séparation des fonctions de propriétaire du réseau. 
d'opérateur, de fournisseur de services, et de fournisseur d'équipements terminaux ; ces différentes fonctions tendent maintenant à devenir des centres de profit opérationnels. Une telle séparation est généralement justifiée pour des raisons économiques, mais on peut douter que la déintégration des GST accroisse leur efficacité. Le fait que les différentes parties du système, qui restent interdépendantes, poursuivent désormais chacune, et de manière indépendante, son intérêt particulier, peut générer des dysfonctionnements. Tel pourrait bien être le résultat de la séparation financière et organisationnelle des services de transport et de la maintenance technique au sein du système de transport aérien. Quant aux conséquences de la séparation entre la gestion des infrastructures et la circulation des trains dans le chemin de fer, elles restent à étudier.

J'ai indiqué que certaines propriétés techniques des premiers GST ont favorisé des formes centralisées d'organisation. Ceci était probablement le plus évident dans les premiers systèmes de télécommunications et pour l'informatique. Dans ces deux domaines, qui ont largement fusionné depuis, les innovations technologiques récentes ont plutôt encouragé la décentralisation. Un micro-ordina- teur actuel, par exemple, a une capacité supérieure à celle d'un ordinateur central d'il y a trente ans. Les développements technologiques dans les télécommunications et l'informatique ont rendu possible la décentralisation, si bien qu'il y a aujourd'hui un changement assez universel de l'ancien paradigme (organisations centralisés et verticalement intégrées) vers un nouveau paradigme postérieur à la déréglementation et préconisant des organisations décentralisées (Genschel \& Werle 1994). Les nouveaux systèmes de télécommunications, à leur tour, rendent possible la dé-hiérarchisation, la décentralisation géographique et la gestion de réseaux dans de nombreux domaines, notamment l'économie. Il semble, encore une fois, que le développement technologique et les changements dans la gestion de la société s'accordent et se renforcent mutuellement. Mais le mouvement dialectique de l'histoire ne s'arrêtera pas à ce stade. Les réseaux en tant que forme d'organisation et de gestion de la société contiennent de nouveaux dangers qui deviendront plus évidents avec le temps. Comme le dit une chanson célèbre, "l'avenir ne nous est pas visible", mais on peut du moins suggérer que la coévolution de la technologie et la gestion de la société continuera.

BIBLIOGRAPHIE

Beniger J. The Control Revolution. Cambridge, Mass: Harvard University Press, 1986.

Braun I., Joerges B. "How to Recombine Large Technical Systems: The Case of European Organ Transplantation." In Changing Large Technical Systems, J. Summerton (ed.). Boulder: Westview Press, 1994, pp. 25-51.

Genschel. P., Werle R. "From National Hierarchies to International Standardization. Modal Changes in the Governance of Telecommunications." Journal of Public Policy 13: 1994, pp. 203-225.

Hohn H.-W., SCHNEIDER V. "Path Dependency and Critical Mass in the Development of Research and Technology." Science and Public Policy, 18, 1991, pp. 111-122.

Hughes T. P. Networks of Power: Electrification in Western Society 1880-1930. Baltimore, Maryland: Johns Hopkins, 1983.

Hughes T. P. Die Erfindung Amerikas. Der technologische Aufstieg der USA seit 1870. Munchen: C.H Beck, 1991.

LA PORTE T. R. Social Responses to Large Technical Systems. Control or Anticipation. Dordrecht: Kluwer Academic Publishers, 1991.

Mayntz R., ScharpF F. W., eds. Gesellschaftliche Selbstregelung und politische Steuerung. Frankfurt a. M.: Campus, 1995.

MAYNTZ R. "Grosstechnische Systeme und ihre gesellschaftstheoretische Bedeutung." Kolner Zeitschrift fur Soziologie und Sozialpsychologie, 45, 1993, pp. 97-108.

Mayntz R., Hughes T. P., eds. The Development of Large Technical Systems. Frankfurt a. M.: Campus, 1988.

RESCH R. Organisationsprobleme der Flugsicherung in Europa. Dissertation, Koln, 1994.

Rochlin G. "Broken Plowshare: System Failure and the Nuclear Power Industry." In Changing Large Technical Systems, J. Summerton (ed.). Boulder: Westview Press, 1994, pp. 231-264

SCHNEIDER V. Institutionelle Evolution als politischer Prozess: Die Entwicklung der Telekommunikation im historischen und internationalen Vergleich. Habilitationsschrift, Koln (Manuskript), 1995.

Schneider V., Thomas G., Vedel T. et al. "The Dynamics of Videotex Development in Britain, France and Germany: A Cross-national Comparison." European Journal of Communication, 6, 1991, pp. 187-212.

Thомаs F. Telefonieren in Deutschland. Organisatorische technische und raumliche Entwicklung eines grosstechnischen Systems. Frankfurt a. M.: Campus, 1995.

Trist E. L., Bamforth K.W. "Some Social and Psychological Consequences of the Longwall Method of Coal-Getting." Human Relations 4, 1951, pp. 3-38.

Trist E. L., Higgins G.W., Murray H., Pollock A.B. Organizational Choice Capabilities of Groups at the Coal Phase under Changing Technologies. London: Tavistock, 1963. 